\title{
Percutaneous Fixation of Proximal Humeral Fractures
}

\author{
Leslie Fink Barnes, MD, Bradford O. Parsons, MD, and Evan L. Flatow, MD \\ Based on an original article: J Bone Joint Surg Am. 2012 Jul 3;94(13):1223-8.
}

\section{Introduction}

The percutaneous approach to proximal humeral fixation emphasizes minimum dissection with the goal of preserving vascularity of the articular segments and thereby decreasing the risk of osteonecrosis.

Proximal humeral fractures vary with regard to pattern, degree of comminution, and patient factors such as age, bone quality, and activity level. These fractures have been treated with interfragmentary suture fixation, percutaneous pinning, plate fixation, intramedullary nailing, hemiarthroplasty, and reverse total shoulder arthroplasty. With any humeral-head-preserving technique, nonunion, malunion, and osteonecrosis are important postoperative concerns.

Previous studies have suggested that percutaneous fixation may decrease the risk of osteonecrosis in patients who have sustained a proximal humeral fracture. The prevalence of osteonecrosis after percutaneous pinning has been reported to be $4 \%$ to $16 \%^{1}$, which is lower than the prevalence of $12.5 \%$ to $71 \%$ reported after the use of other open techniques. Therefore, percutaneous pinning substantially reduces, although does not completely eliminate, the risk of the poor clinical outcomes seen in patients who develop osteonecrosis.

Percutaneous fixation of proximal humeral fractures involves careful patient selection and appropriate timing of the fixation. The procedure begins with an understanding of the fracture fragments and deforming forces, as the minimally invasive technique does not allow direct inspection. The most common fracture pattern treated with this technique consists of a humeral head fragment posterior to the humeral shaft and in valgus alignment. The greater tuberosity fragment is pulled posterosuperiorly by the insertion of the rotator cuff, while the lesser tuberosity is pulled medially.

The initial reduction maneuvers are designed to lever the humeral head up and anteriorly from its posteriorly impacted position to realign it with the humeral shaft; this is facilitated by the use of a percutaneous elevator with fluoroscopic guidance. Terminally threaded pins are then inserted percutaneously to secure this reduction. Orthogonal images are essential to confirm that pins have not penetrated the joint. With use of a bone hook, the greater tuberosity is then reduced to the humeral head percutaneously with a neutralizing force, after which it is fixed securely with cannulated screws under fluoroscopic guidance. The lesser tuberosity is then reduced and fixed with terminally threaded pins or cannulated screws if indicated.

The operative technique for percutaneous fixation of proximal humeral fractures involves the following steps.

\section{Step 1: Preoperative Planning}

Perform preoperative imaging to assess fracture displacement and comminution in order to confirm that percutaneous treatment is appropriate.

- Obtain preoperative true anteroposterior (Grashey) and axillary radiographs (Fig. 1), and a computed tomography (CT) scan of the affected shoulder, to assess fracture displacement and comminution in order to confirm that percutaneous treatment is appropriate.

- We recommend performing surgery within seven to ten days after the injury, as extensive fracture mobilization is not easily achieved with a percutaneous technique in the presence of callus formation.

\section{Step 2: Closed Fracture Reduction}

Attempt closed fracture reduction prior to incision.

- Position the patient in the modified beach-chair position or supine with an access route for a standard-size fluoroscopy unit to obtain orthogonal imaging (Figs. 2-A through 2-D).

- For anesthesia, we prefer a regional technique with a peripheral nerve catheter in addition to general anesthesia with a laryngeal mask airway.

- In the operating room, perform a reduction maneuver with fluoroscopic guidance before 
preparing and draping the patient to ensure that closed reduction and orthogonal radiographs can be obtained.

- Reduce the fracture with progressive longitudinal traction with the shoulder abducted to $70^{\circ}$ to $80^{\circ 2}$.

- Exert manual posterior pressure on the shaft to counteract the typical anterior displacement.

- Place the arm in a pneumatic arm holder.

\section{Step 3: Surgical Approach for Humeral} Head and Shaft Reduction

Obtain anatomic humeral head reduction, insert terminally threaded pins, and check pin placement fluoroscopically.

- When anatomic reduction cannot be obtained with a closed reduction maneuver, make a $1-\mathrm{cm}$ longitudinal incision over the proximal lateral part of the deltoid to first reduce the surgical neck fracture. This reduction portal is located inferior to the anterolateral corner of the acromion at the level of the surgical neck and must be $>5 \mathrm{~cm}$ distal to the acromion along the lateral aspect of the humeral shaft to avoid the anterior branch of the axillary nerve ${ }^{3}$ (Fig. 3).

- Introduce a blunt elevator or bone tamp to maneuver the head out of valgus (Video 1), and confirm the position on fluoroscopy ${ }^{4}$. Place the tamp carefully at the posterolateral cortex of the humeral head and angle it proximally toward the glenoid, subtending an angle of approximately $20^{\circ}$ relative to the longitudinal line of the humeral shaft. This will help avoid destruction of the humeral head during reduction (Figs. 4, 5-A, and 5-B).

- Make every effort to restore the anatomic position of the humeral head in relation to the humeral shaft, with restoration of continuity along the medial calcar (also known as the "Gothic arch" of the shoulder [Fig. 6-A]). Avoid valgus. The head should be within $10^{\circ}$ of normal version with $<10 \%$ translation.

- After adequate fracture reduction is confirmed on anteroposterior and axillary radiographs, place two 2.8-mm terminally threaded pins in a retrograde fashion through two percutaneous stab incisions on the anterior and lateral aspects of the humeral shaft ${ }^{5}$ (Video 2). Confirm the trajectory of the pins with fluoroscopy by first overlaying the threaded pins exteriorly.

- Make two stab incisions over the lateral aspect of the arm just proximal to the deltoid insertion (about twice the distance from the acromion as the incision for the fracture reduction) for percutaneous pin placement (Fig. 3). After skin incision, dissect bluntly using a pointed-tip instrument, such as a clamp, to spread through soft tissue.

- Using a soft-tissue protector, from entry points distal to the fracture line as described above, start inserting the pins perpendicular to the shaft and then tilt and aim them both proximally $45^{\circ}$ relative to the perpendicular in the scapular plane and posteriorly $30^{\circ}$ in the coronal plane to advance them across the fracture line (Fig. 6-A).

- Advance the Kirschner wires with a power driver until all but the terminal $5 \mathrm{~mm}$ have been inserted and then advance them by hand, using a T-handle chuck, to the subchondral surface. We have found that a T-handle chuck provides more tactile sensation than a power drill, allowing us to avoid intra-articular penetration.

- Check the final position of the pins fluoroscopically in orthogonal planes to confirm extraarticular placement. With both pins crossing the fracture site, gentle rotation of the humerus should not result in loss of reduction (Figs. 6-A and 6-B).

- If the articular surface is penetrated by the pins, reposition them in a different track. Otherwise, they can migrate and penetrate the articular surface.

- Cut the pins and bury them deeply beneath the skin to minimize the risk of pin-track infection. The pins will be removed, three to four weeks later, in the operating room.

\section{Step 4: Tuberosity Fracture Reduction and Fixation of the Tuberosity Fragments}

Fix the tuberosity fracture(s).

- Free the tuberosity fragments using a Cobb elevator for mobilization and reduce them using a dental pick or bone hook percutaneously through the original anterolateral incision. The arm remains in neutral rotation (Fig. 7).

- Reduce the greater tuberosity to, or to no more than $5 \mathrm{~mm}$ below, the articular surface of the humeral head to restore rotator cuffdynamics. The tuberosity fragments are ultimately secured with cannulated small-fragment screws or with pins that are then cut below the skin (Video 3).

- Make a stab incision at the level of the greater tuberosity and insert the drill sleeve with the Kirschner wire in place as an obturator (Figs. 8-A and 8-B).

- A wide spread of the pins within the head ensures the stability of the fixation. The optimal trajectories of the threaded pins are shown in Figures 9-A and 9-B. 
- A 3.0-mm Schanz pin can be inserted as a joystick into the free fragment of the greater tuberosity and then, after the tuberosity has been maneuvered into position with the Schanz pin, the pin can be advanced as provisional fixation across the fracture site.

- $\quad$ Place the guide pins for 4.5-mm cannulated screws. Place the distal perpendicular pin first, and then insert a diagonal pin through a more proximal portion of the tuberosity, aiming toward the medial calcar (Fig. 9-B). Placing the perpendicular screw first helps prevent overreduction of the greater tuberosity downward. Then insert the 4.5-mm cannulated screws over the guide pins through the drill sleeve. Be sure to penetrate the medial aspect of the shaft with the diagonal screw.

- After fixation of the greater tuberosity, turn your attention to the lesser tuberosity. Fixation of the lesser tuberosity can be controversial, as it is often stable with the piece of humeral head. Check under continuous fluoroscopy for movement of the lesser tuberosity with internal and external rotation of the arm.

- If the lesser tuberosity needs to be fixed, use unicortical cannulated screws for fragment fixation by inserting guide pins from anterior to posterior followed by the cannulated screw (Video 4). The arm remains in neutral position (Fig. 10).

- The starting point for the anterior Kirschner wires should be lateral to the coracoid in order to capture the fragment without risking damage to local neurovascular structures.

- If the lesser tuberosity is substantially displaced medially, make an incision through the skin that is just large enough for an index finger. Spread with a blunt clamp to the bone of the humeral head, then manually free the fragment from soft tissue.

- Advance the anterior Kirschner wire under fluoroscopic guidance into the lesser tuberosity fragment and use it as a joystick to manipulate the piece into the proper position.

- With the lesser tuberosity fracture reduced, advance the Kirschner wire and insert a cannulated screw over the wire.

\section{Step 5: Wound Closure}

Cut the pins below the skin and irrigate and close the wounds.

- After confirmation of fracture reduction and appropriate pin and screw placement with orthogonal fluoroscopic images, cut the terminally threaded pins below the skin using a wire cutter.

- Be sure that the fracture is stable with gentle internal and external rotation under fluoroscopy.

- Remove the remaining guide pins used during cannulated screw placement.

- Irrigate the wounds thoroughly.

- Close the wounds with buried 3-0 absorbable Monocryl (poliglecaprone; Ethicon, Johnson \& Johnson) suture. Apply Steri-Strips (3M) and gauze bandages.

\section{Step 6: Postoperative Rehabilitation}

Begin passive shoulder motion after pin removal, and initiate active motion at six weeks after pin removal.

- Patients wear a sling for shoulder immobilization for three to four weeks. No motion is allowed until pin removal.

- Remove the pins, with local anesthesia and fluoroscopic guidance, in the operating room three to four weeks postoperatively. While the patient is under anesthesia, evaluate the range of motion and perform a gentle manipulation if indicated.

- Protected, passive shoulder range-of-motion exercises, including forward elevation and external rotation with the arm at the side as well as pendulum exercises, are begun following removal of the pins.

- Active motion is initiated at six weeks after pin removal, provided that healing is demonstrated on postoperative radiographs.

- Strengthening starts at two to three months after pin removal as tolerated.

\section{Results}

The results of this technique in twenty-seven patients with an average age of 58.8 years (range, forty-two to seventy-six years) at the time of injury were reported previously ${ }^{6}$. There were five two-part fractures, twelve three-part fractures, and ten four-part fractures. All ten of the four-part fractures demonstrated a valgus-impacted pattern. The average duration of follow-up was eightyfour months.

No patient had nonunion or failure, loosening, or complications related to the implant. The average American Shoulder and Elbow Surgeons (ASES) score for all patients followed for a minimum of three years was 82 . The patients without osteonecrosis had, on the average, $144^{\circ}$ of forward elevation, $44^{\circ}$ of external rotation, and internal rotation to L2. The ASES scores for the patients without posttraumatic osteoarthritis averaged 87 compared with 74 for those with posttraumatic 
osteoarthritis prior to arthroplasty, which was a significant difference $(p=0.046)$.

Osteonecrosis occurred in seven (26\%) of the twenty-seven patients; three additional patients $(11 \%)$ also had radiographic evidence of posttraumatic arthritis. Osteonecrosis was diagnosed, on the average, fifty months after the original percutaneous fixation. The highest rate of osteonecrosis was seen in the patients with a four-part fracture, with five of the ten developing osteonecrosis. Patients with symptomatic osteonecrosis had significantly less forward elevation $\left(125^{\circ} ; p=0.01\right)$ and external rotation $\left(23^{\circ} ; p=0.02\right)$, whereas internal rotation was to L2. Of the seven patients with osteonecrosis, two were symptomatic; one underwent revision to a hemiarthroplasty at twenty-one months after surgery and the other, at one year.

\section{What to Watch For}

\section{Indications}

- Proximal humeral fractures with angulation at the surgical neck of $>45^{\circ}$ or $>1 \mathrm{~cm}$ of displacement ( $>5 \mathrm{~mm}$ of displacement for greater tuberosity fragments) in the absence of substantial comminution or a head-split fracture.

- Valgus-impacted four-part fractures are the most common indication, but this technique can be performed for selected two, three, or four-part fractures; three-part fractures are often the most challenging.

- Isolated surgical neck fractures are the most straightforward to manage.

\section{Contraindications}

- Substantial comminution in the humeral head or calcar region.

- Severely displaced or irreducible three and fourpart fractures and fracture-dislocations.

- Bone loss or osteopenia.

- Varus fractures.

- A delay of more than ten days from the injury.

\section{Pitfalls \& Challenges}

- Reduction can be difficult with this limited incision technique.

- The trajectory and starting point of the terminally threaded pins must avoid the axillary nerve.

- Pins that penetrate the articular surface cannot be backed out and left in the same track as they can advance and migrate.

- Each pin should enter the arm above the insertion of the deltoid to avoid the radial nerve in the spiral groove.

- We recommend using 2.8-mm terminally threaded pins for optimal fixation.

- Osteonecrosis is an important postoperative concern with any humeral-head-preserving technique.

- If humeral head replacement is required for osteonecrosis after percutaneous pinning, it is not much more complex than a primary arthroplasty as there is little residual hardware. This is in contrast to a replacement done in a patient with a prior open reduction and internal fixation with a plate and screws.

- $\quad$ Pin-track infections can be minimized by burying the pins deep under the skin. If a pin backs out, it should be removed and any infection should be treated with oral antibiotics.

\section{Clinical Comments}

What measures have you found to be effective in reducing the risk of complications after surgery for proximal humeral fractures?

Cutting the pins as short as possible, deep under the skin, avoids skin irritation and pin-track infection although it does require a second trip to the operating room for removal. Appropriate rehabilitation is also essential for avoiding complications.

How do you manage postoperative complications?

Loss of fixation is a possible early complication, especially if a patient is reinjured or is unable to tolerate immobilization in the sling. If displacement is substantial, open reduction and revision fixation may be required. Stiffness is another possible complication and is managed with gentle manipulation at the time of pin removal or with arthroscopic capsular release. The most common late postoperative complication is osteonecrosis. Therefore, we counsel patients about this risk preoperatively, and follow them for several years postoperatively if there is any concern. Removal of hardware or arthroplasty is the recommended treatment for symptomatic posttraumatic osteonecrosis.

What are important factors to consider preoperatively?

The most appropriate case for this technique is a valgus-impacted fracture without substantial comminution in a younger patient without a substantial delay between the injury and surgical treatment. required?

How often is fixation of the lesser tuberosity

If the head is tilted up but retroversion is not restored, iatrogenic displacement of the lesser tuberosity results. Placing an elevator anterolaterally to drive the head out of valgus and also out of anteversion reduces the need to manipulate the lesser tuberosity. However, in many cases, reduction of the lesser tuberosity is necessary. 
Disclosure: None of the authors received payments or services, either directly or indirectly (i.e., via his or her institution), from a third party in support of any aspect of this work. One or more of the authors, or his or her institution, has had a financial relationship, in the thirty-six months prior to submission of this work, with an entity in the biomedical arena that could be perceived to influence or have the potential to influence what is written in this work. In addition, one or more of the authors has a patent or patents, planned, pending, or issued, that is broadly relevant to the work. No author has had any other relationships, or has engaged in any other activities, that could be perceived to influence or have the potential to influence what is written in this work. The complete Disclosures of Potential Conflicts of Interest submitted by authors are always provided with the online version of the article.

\section{References}

1. Wijgman AJ, Roolker W, Patt TW, Raaymakers EL, Marti RK. Open reduction and internal fixation of three and four-part fractures of the proximal part of the humerus. J Bone Joint Surg Am. 2002 Nov;84(11):1919-25.

2. Jaberg H, Warner JJ, Jakob RP. Percutaneous stabilization of unstable fractures of the humerus. J Bone Joint Surg Am. 1992 Apr;74(4):508-15.

3. Flatow EL, Cuomo F, Maday MG, Miller SR, Mcllveen SJ, Bigliani LU. Open reduction and internal fixation of two-part displaced fractures of the greater tuberosity of the proximal part of the humerus. J Bone Joint Surg Am. 1991 Sep;73(8):1213-8.

4. Resch H, Beck E, Bayley I. Reconstruction of the valgus-impacted humeral head fracture. J Shoulder Elbow Surg. 1995 MarApr;4(2):73-80.

5. Keener JD, Parsons BO, Flatow EL, Rogers K, Williams GR, Galatz LM. Outcomes after percutaneous reduction and fixation of proximal humeral fractures. J Shoulder Elbow Surg. 2007 May-Jun;16(3):330-8. Epub 2007 Feb 22.

6. Harrison AK, Gruson KI, Zmistowski B, Keener J, Galatz L, Williams G, Parsons BO, Flatow EL. Intermediate outcomes following percutaneous fixation of proximal humeral fractures. J Bone Joint Surg Am. 2012 Jul 3;94(13):1223-8.

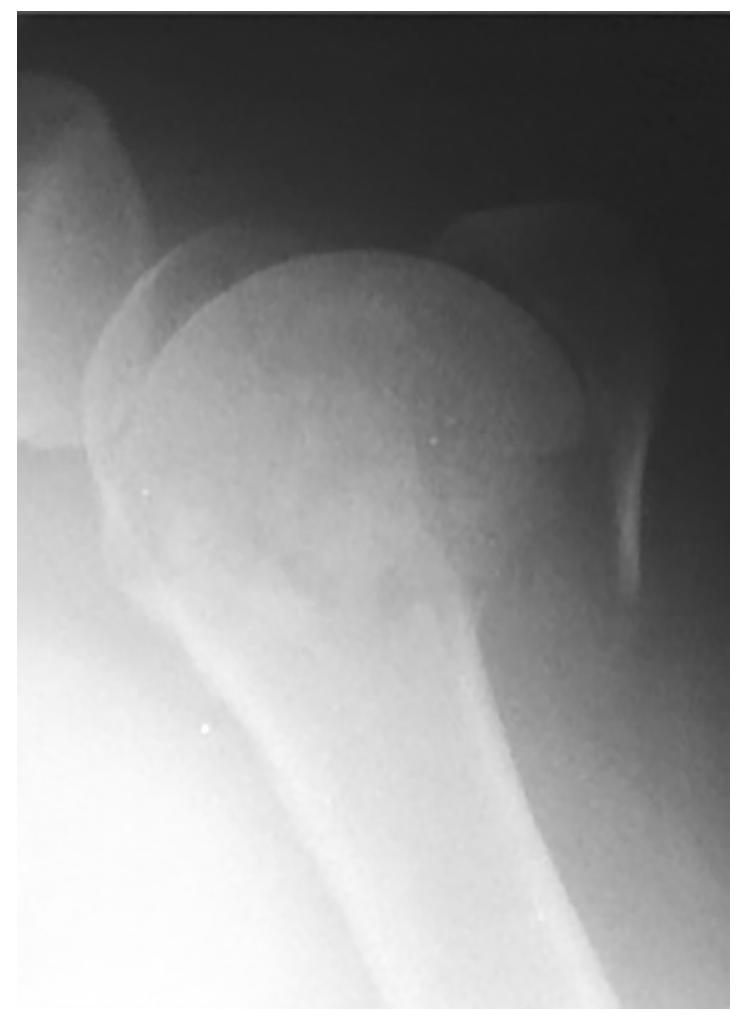

Fig. 1

Preoperative radiograph showing a four-part proximal humeral fracture. 


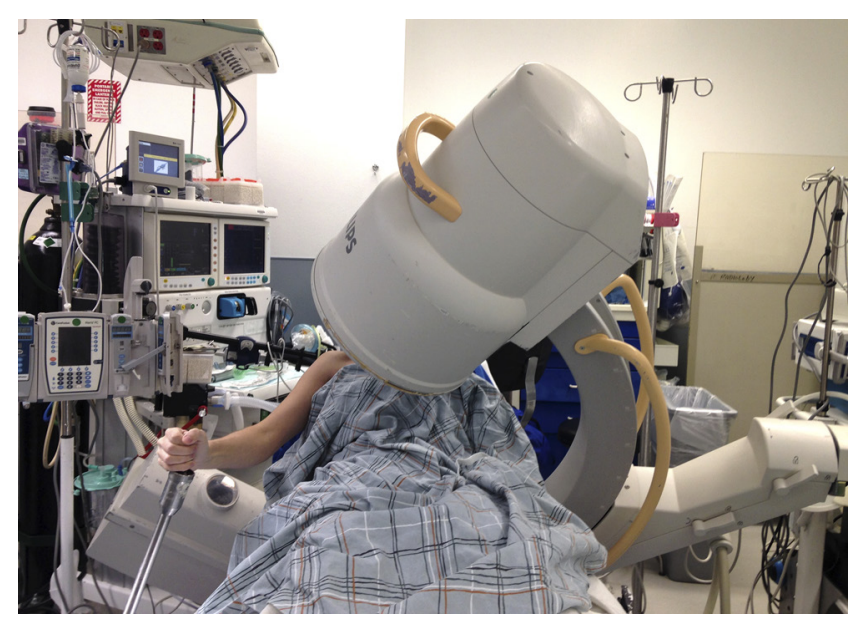

Fig. 2-A

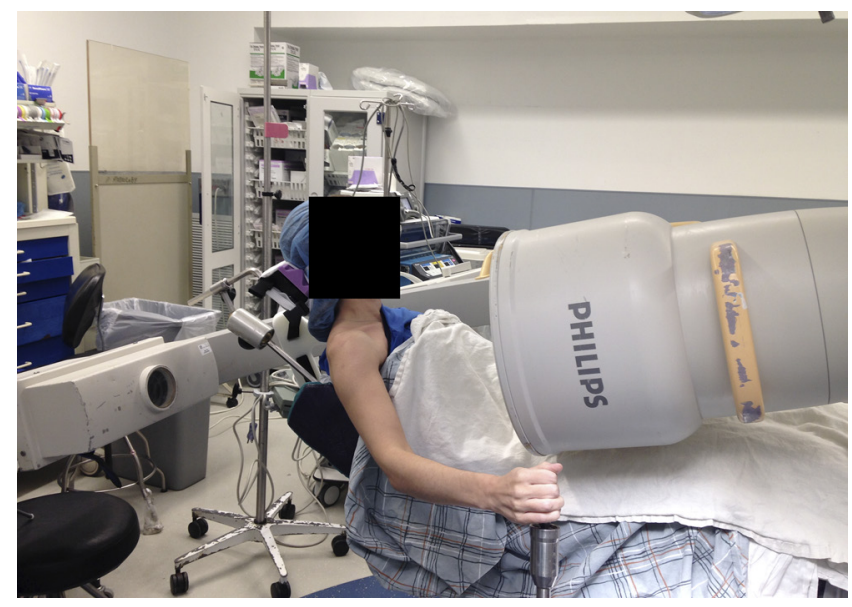

Fig. 2-C

Figs. 2-A through 2-D Surgical setup with beach-chair positioning and fluoroscopic imaging on the contralateral side for best surgical access.

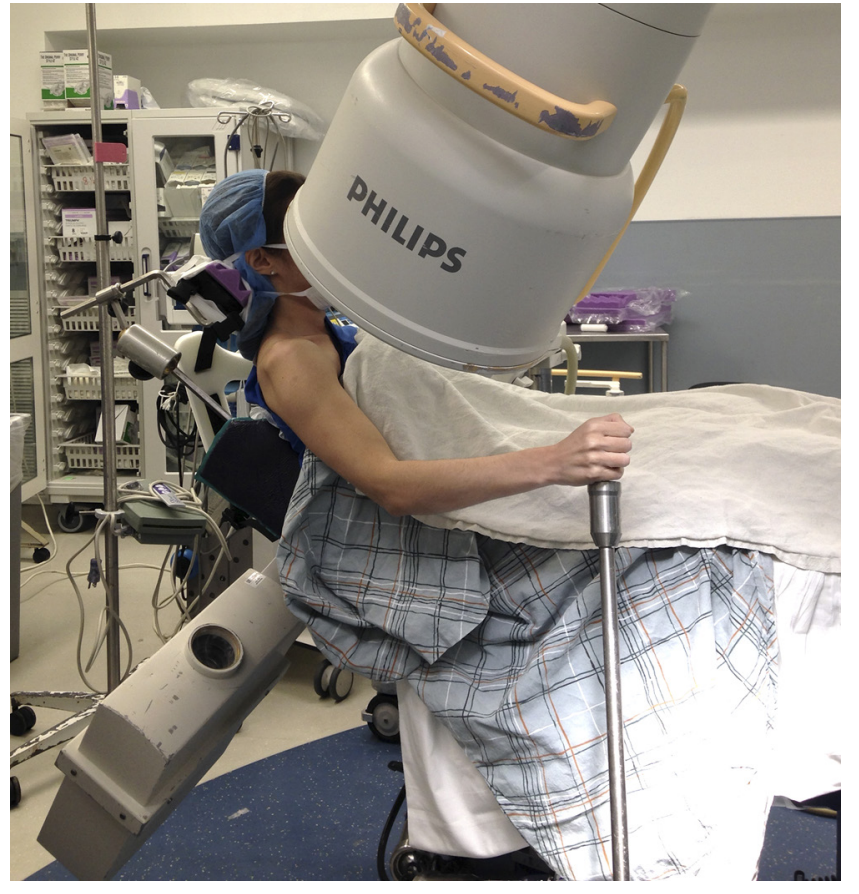

Fig. 2-B

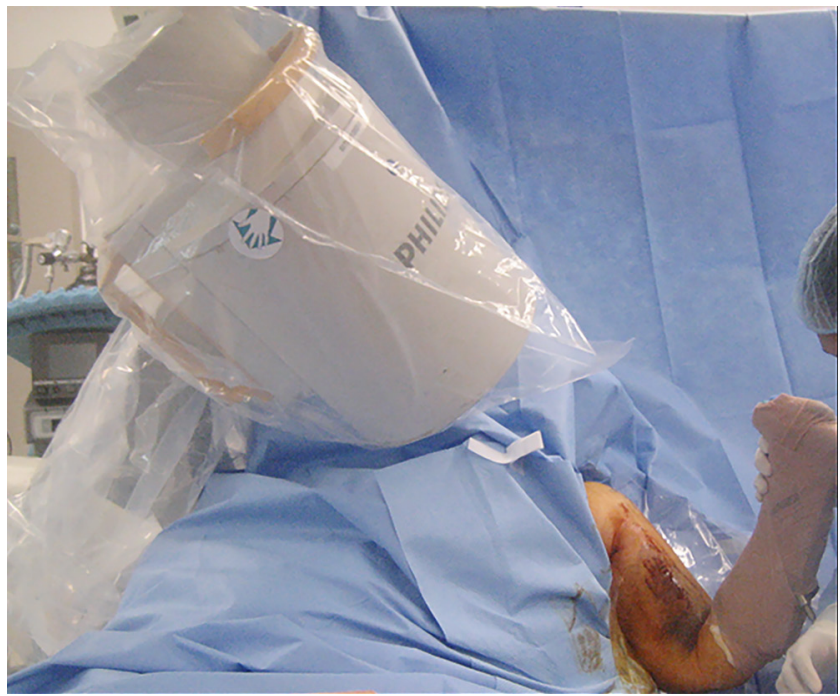

Fig. 2-D 


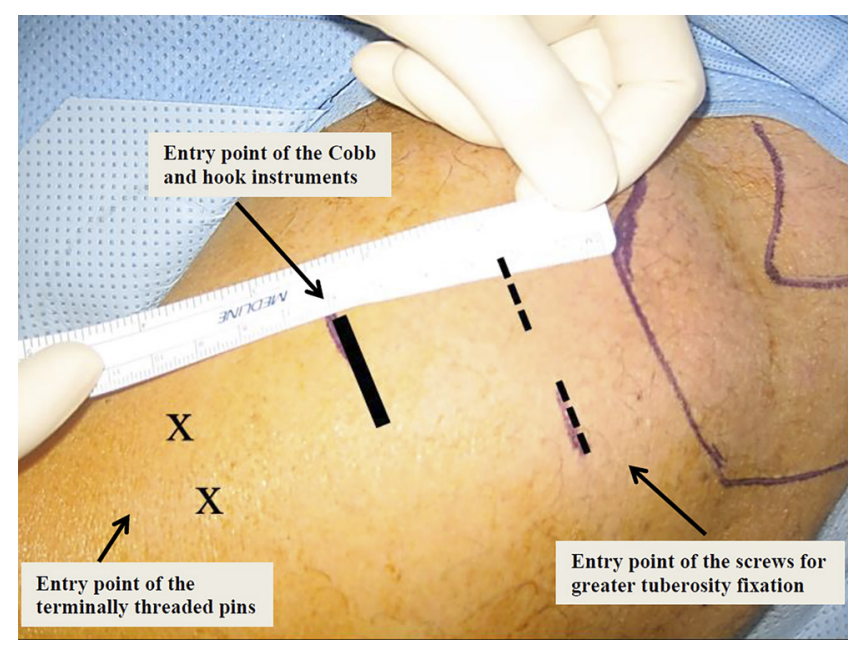

Fig. 3

Location of the skin incisions for the percutaneous technique. The thick line indicates the entry point of the Cobb and hook instruments, the X's mark the entry points of the terminally threaded pins, and the dashed lines indicate the locations of the cannulated screws used to fix the greater tuberosity.

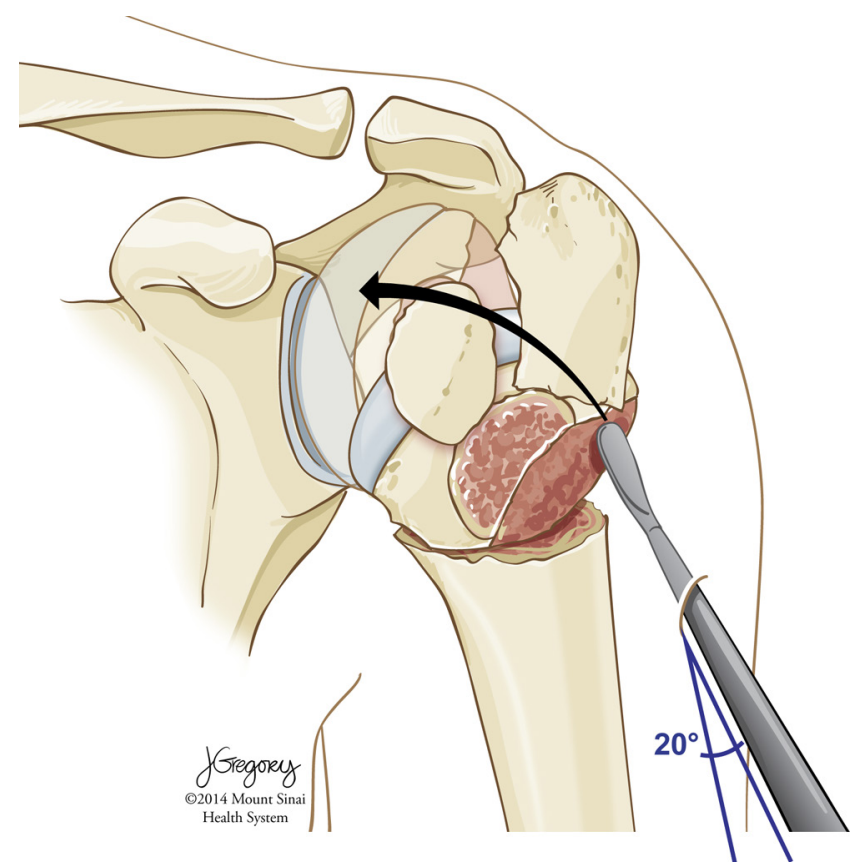

Fig. 4

Drawing of the humeral head fracture reduction maneuver with use of a Cobb instrument. (Reproduced with permission from (C) Mount Sinai Health System. Reproduced with modification.)

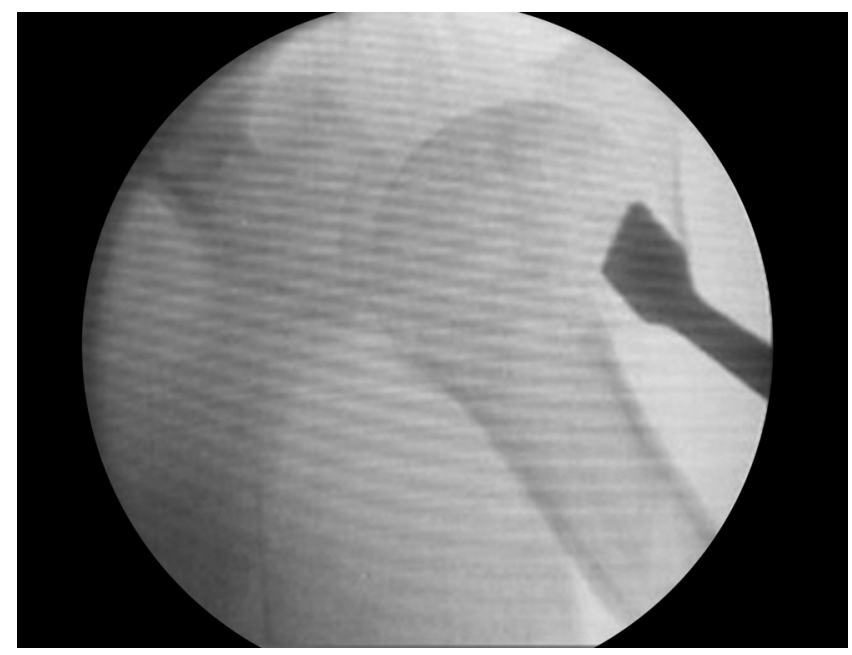

Fig. 5-A

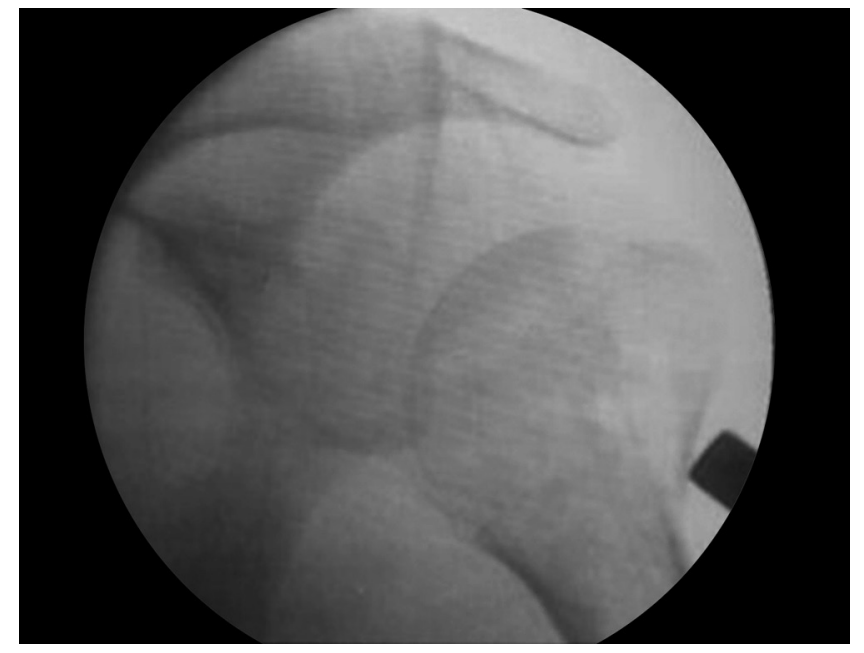

Fig. 5-B

Figs. 5-A and 5-B Intraoperative radiographs showing the Cobb reduction maneuver. 


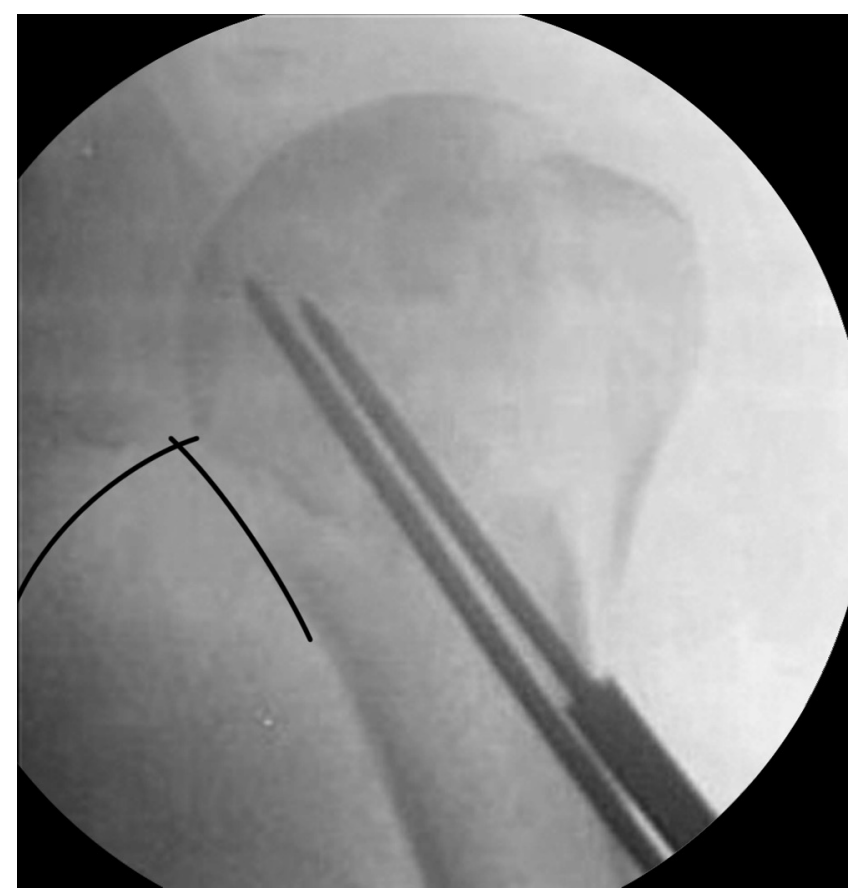

Fig. 6-A

Figs. 6-A and 6-B The humeral head is stabilized to the shaft with terminally threaded pins, and orthogonal radiographs are obtained. The black lines in Fig. 6-A demonstrate restoration of the Gothic arch.

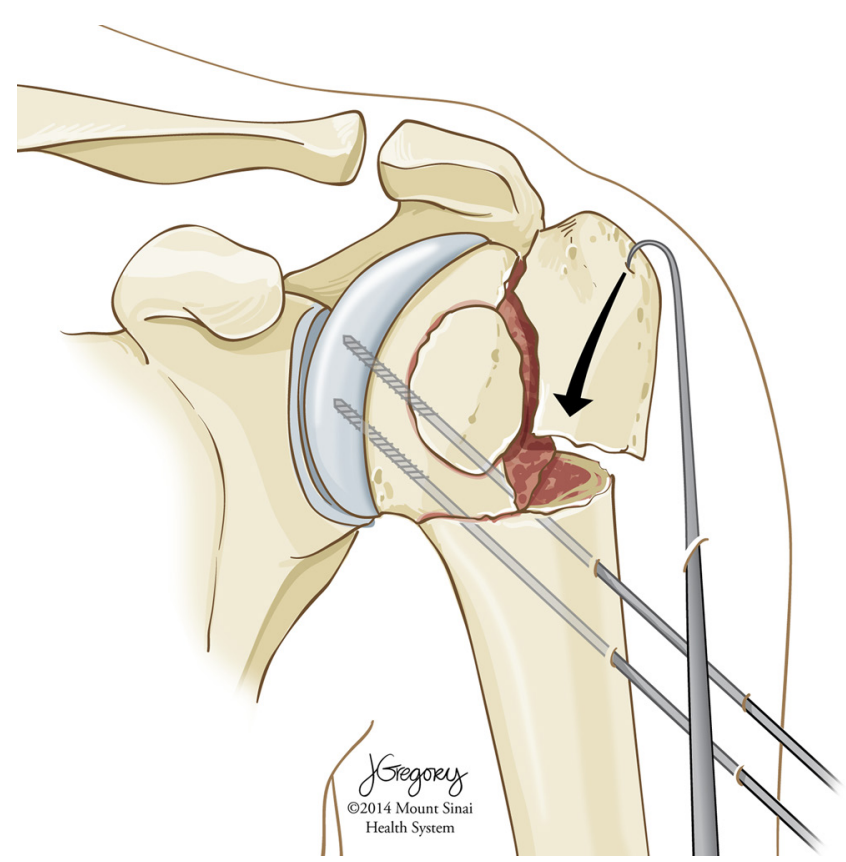

Fig. 7

Reduction of the greater tuberosity fragment with a hook instrument. (Reproduced with permission from @ ( Mount Sinai Health System.)

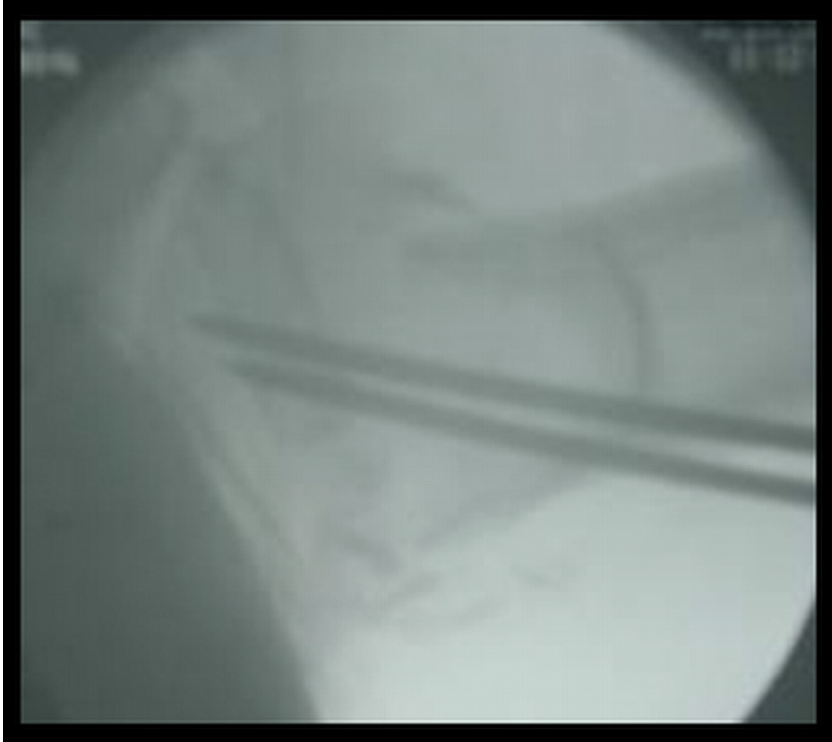

Fig. 6-B 


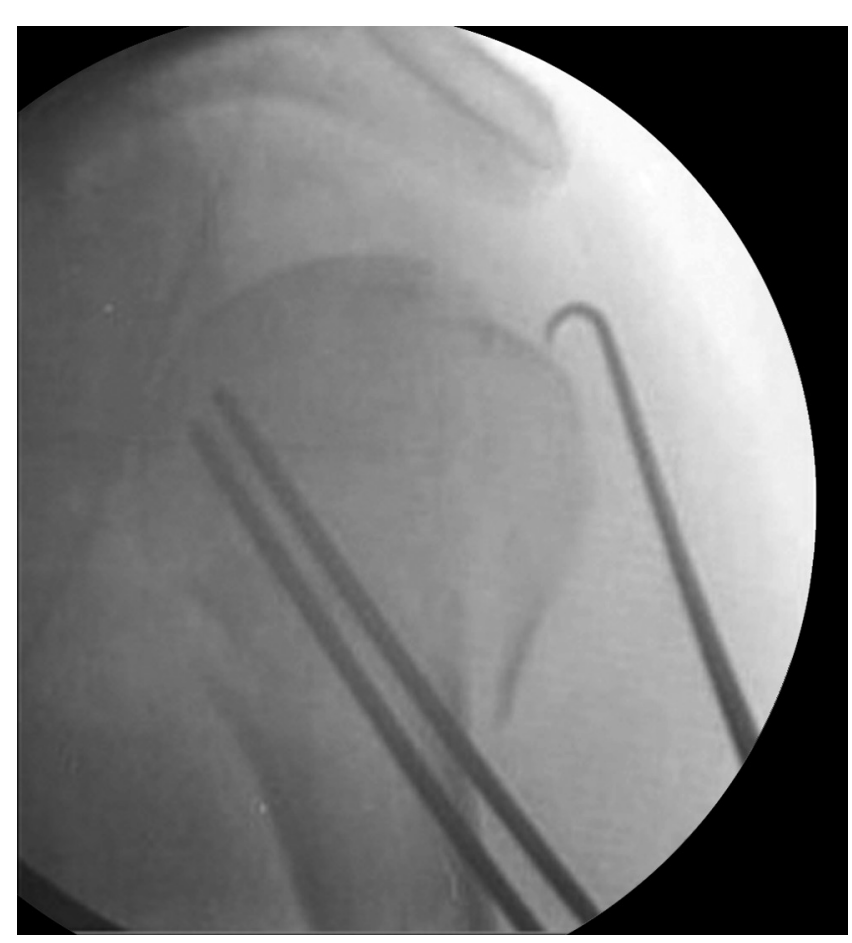

Fig. 8-A

Fig. 8-B

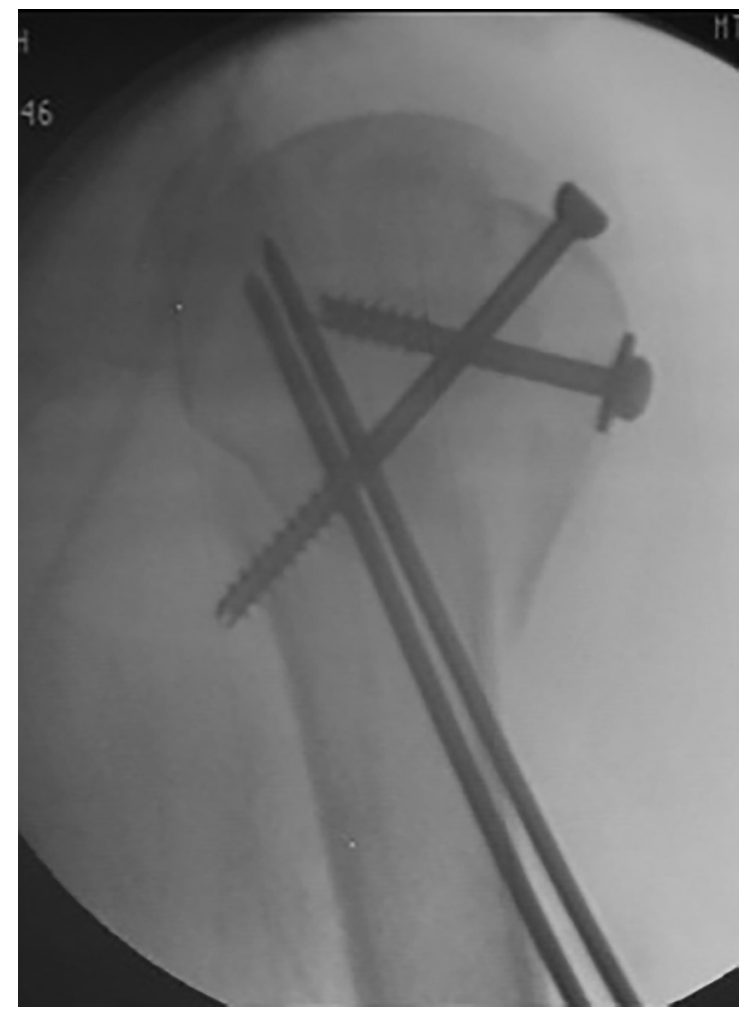

Fig. 9-A

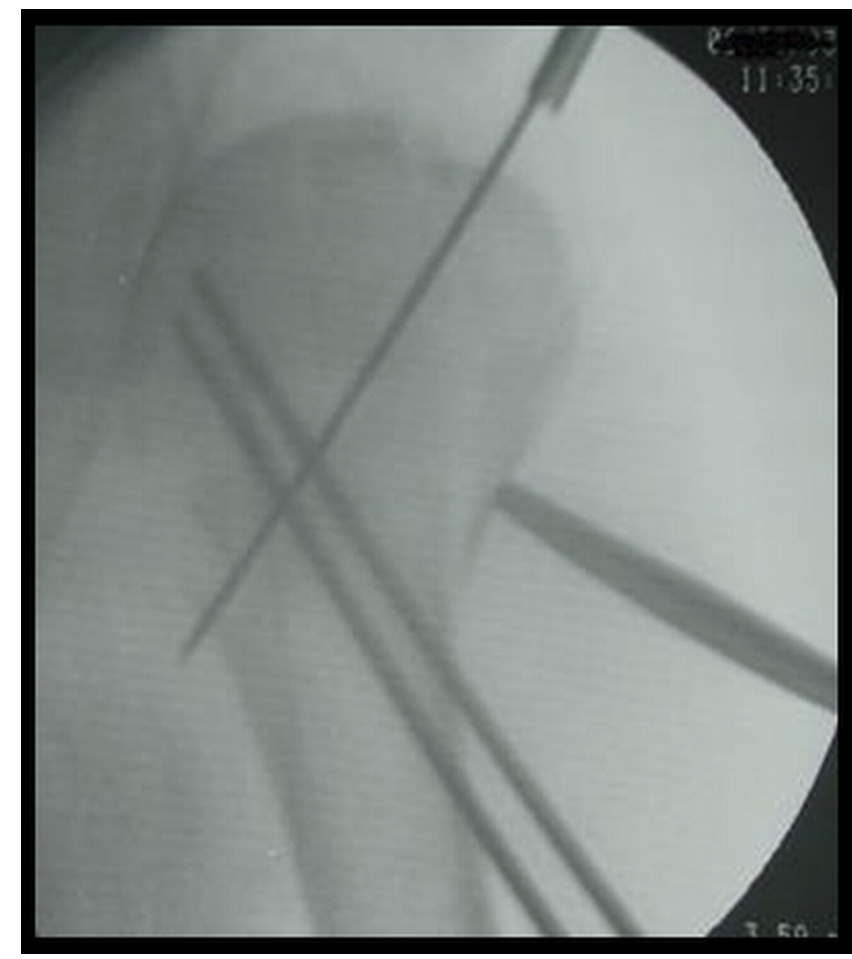

rosity and stabilization of the fragment with cannulated screws.

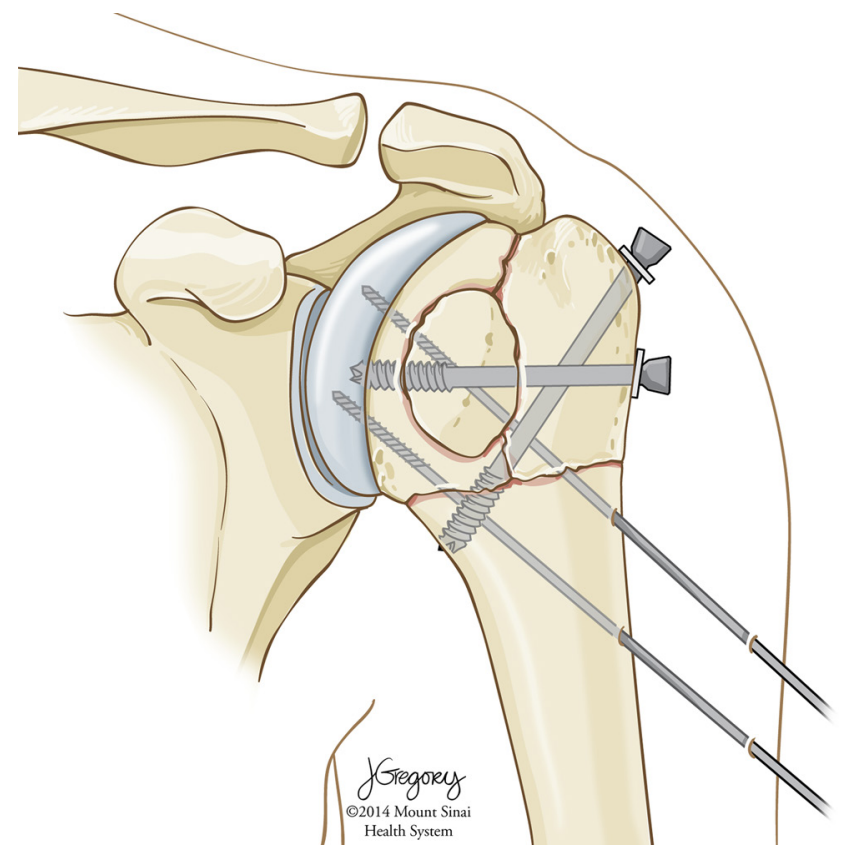

Fig. 9-B

Fig. 9-A Radiograph showing the final pin configuration. Fig. 9-B Drawing showing the final pin configuration. (Reproduced with permission from (c) Mount Sinai Health System.) 


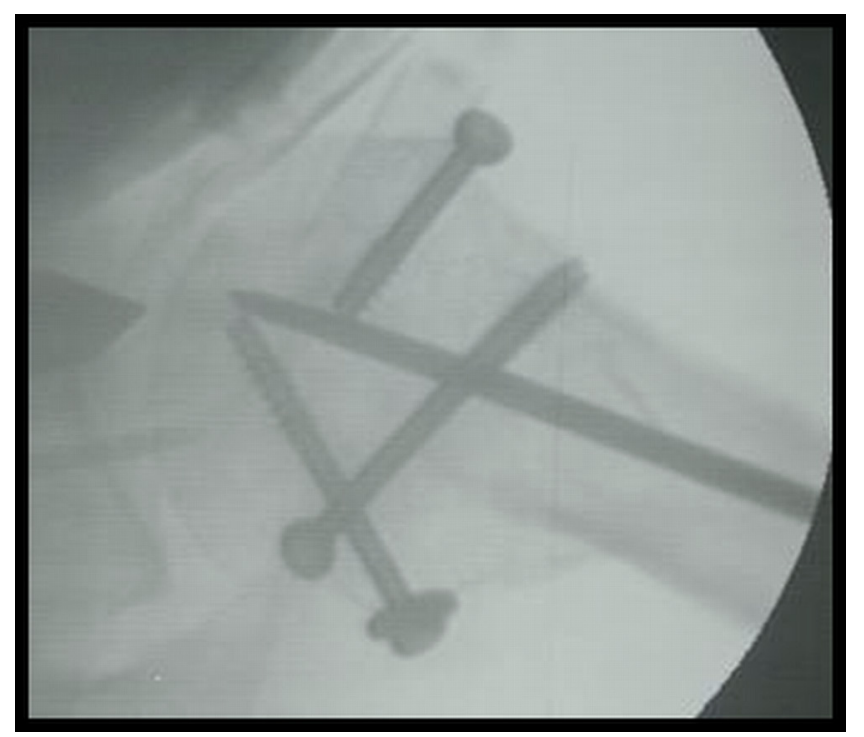

Fig. 10

Axillary radiograph showing the position of the anterior screw if fixation of the lesser tuberosity is required. 